Ana Angélica Bulcão Portela Lindoso 1

Eliseu Alves Waldman"

Naomi Kawaoka Komatsu"I

Sumie Matai de Figueiredo'"I

Mauro Taniguchi"'I

Laura C Rodrigues ${ }^{\mathrm{IV}}$

\section{Perfil de pacientes que evoluem para óbito por tuberculose no município de São Paulo, 2002}

\author{
Profile of tuberculosis patients \\ progressing to death, city of São \\ Paulo, Brazil, 2002
}

\section{RESUMO}

OBJETIVO: Descrever o perfil de pacientes adultos residentes no município de São Paulo que evoluíram para óbito associado à tuberculose, segundo fatores biológicos, ambientais e institucionais.

MÉTODOS: Estudo descritivo, abrangendo todos os óbitos por tuberculose $(\mathrm{N}=416)$ ocorridos em 2002, entre maiores de 15 anos. Os dados analisados foram obtidos do Sistema Municipal de Informações de Mortalidade, prontuários hospitalares, Serviço de Verificação de Óbitos e Sistema de Vigilância de Tuberculose. Os cálculos dos riscos relativos e intervalos de confiança de 95\% (IC 95\%) tiveram como referência o sexo feminino, grupo de 15 a 29 anos, e os naturais do Estado de São Paulo. A análise comparativa usou o teste do qui-quadrado de Pearson e o exato de Fisher para variáveis categóricas e o teste Kruskal-Wallis para variáveis contínuas.

RESULTADOS: Do total de óbitos, 78\% apresentavam a forma pulmonar; o diagnóstico foi efetuado após a morte em $30 \%$ e em unidades de atendimento primário em 14\% dos casos; 44\% não iniciaram tratamento; 49\% não foram notificados; $76 \%$ eram homens e a mediana da idade foi de 51 anos; $52 \%$ tinham até quatro anos de estudo, $4 \%$ eram prováveis moradores de rua. As taxas de mortalidade aumentavam com a idade, sendo de 5,0/100.000 no município, variando de zero a 35, conforme o distrito. Para 82 de 232 pacientes com registro de tratamento, havia referência de tratamento anterior, e desses, 41 o haviam abandonado. Constatou-se presença de diabetes (16\%), doença pulmonar obstrutiva crônica (19\%), HIV (11\%), tabagismo (71\%) e alcoolismo (64\%) nos pacientes.

Município de São Paulo. São Paulo, SP, Brasil

iv London School of Hygiene and Tropical Medicine. London, United Kingdom

Correspondência | Correspondence: Eliseu Alves Waldman

Faculdade de Saúde Pública Universidade de São Paulo

Av. Dr. Arnaldo, 715

01246-904 São Paulo, SP, Brasil

E-mail: eawaldma@usp.br

CONCLUSÕES: Homens acima de 50 anos, migrantes e residentes em distritos com baixo Índice de Desenvolvimento Humano apresentam maiores riscos de óbito. A pouca escolaridade e apresentar co-morbidades são características importantes. Observou-se baixa participação das unidades básicas de saúde no diagnóstico e a elevada sub-notificação.

DESCRITORES: Tuberculose, mortalidade. Comorbidade. Perfil de Saúde. Epidemiologia Descritiva. Brasil. 


\begin{abstract}
OBJECTIVE: To profile adult patients dying of tuberculosis in the city of São Paulo with respect to biological, environmental and institutional factors.

METHODS: Descriptive study covering all tuberculosis deaths ( $\mathrm{N}=416)$ among individuals aged over 15 years in 2002. Data were obtained from hospital records, the local Mortality Information System, Coroner's Service, and tuberculosis Surveillance System. The estimates of relative risk and 95\% confidence intervals (95\% CI) were based on the reference group, i.e., females aged 15 to 29 years, originally from the State of São Paulo (Brazil). A comparative analysis was conducted using Pearson's chi-square test and Fisher's exact test for categorical variables and Kruskal-Wallis test for continuous variables.
\end{abstract}

RESULTS: Of all tuberculosis deaths identified, 78\% had pulmonary form. Tuberculosis diagnosis was made after death in $30 \%$ and in primary health care units in $14 \%$. Of them, $44 \%$ had not started treatment; $49 \%$ were not notified; and $76 \%$ were men. The median age was 51 years; $52 \%$ had up to four years of schooling; $4 \%$ were probably living in the streets. Mortality rate increased with age; it was 5.0/100,000 for the entire city, ranging between zero to 35 according to the district. Previous treatment was reported for 82 out of 232 patients, and of them, 41 defaulted treatment. Diabetes (16\%), chronic obstructive pulmonary disease (19\%), HIV infection (11\%), smoking (71\%), and alcohol abuse (64\%) were also reported.

CONCLUSIONS: Adult males over 50, migrants and living in lower Human Development Index districts were more likely to die of tuberculosis. Low schooling and comorbidities are relevant characteristics. Low involvement of primary care units in tuberculosis diagnosis and high underreporting of cases were also seen.

DESCRIPTORS: Tuberculosis, mortality. Comorbidity. Health Profile. Epidemiology, Descriptive. Brazil.

\title{
INTRODUÇÃO
}

O Brasil situa-se entre os países com maiores taxas de morbi-mortalidade por tuberculose (TB), tendo registrado anualmente cerca de 85.000 casos novos e 6.000 óbitos. ${ }^{6}$ De 1980 a 1995, a proporção de óbitos associados à TB elevou-se de 10,1\% para 15,5\%, em relação ao total de mortes por doenças transmissíveis (Capítulo I) da Classificação Internacional Estatística de Doenças (CID-10) ocorridos no País. ${ }^{13}$ Tais dados apontam o elevado nível endêmico da TB e uma tendência distinta da maioria das doenças infecciosas, cuja incidência diminuiu de forma expressiva nas últimas duas décadas. ${ }^{4, a}$

No município de São Paulo, as taxas de morbi-mortalidade por TB aproximam-se da média registrada no País, sendo, no entanto, mais elevadas do que as registradas no interior do estado (Galesi, 1998). ${ }^{\mathrm{b}}$ Em 2000, esse município apresentou taxa de incidência de 65/100.000 habitantes, com $60 \%$ dos casos novos bacilíferos, e a taxa de mortalidade em 5,7/100.000 habitantes. Essas taxas variam quando analisadas por distritos, com incidência entre 18,7 e 261,1/100.000 habitantes. Ausente em alguns distritos, a mortalidade alcançava taxas de até 20,9/100.000 habitantes naqueles aonde residem os segmentos mais pobres da população.c

Alguns dados disponíveis sobre as atividades de controle da TB na capital são consistentes com a grave situação dessa endemia. A proporção de curas situavase, em 2000 , em torno de $70 \%$, o abandono em $20 \%$ e a letalidade em $13 \%{ }^{c}$

O objetivo do presente estudo foi descrever o perfil de pacientes adultos residentes no município de São Paulo que evoluíram para óbito associado à tuberculose, segundo fatores biológicos, ambientais e institucionais.

\footnotetext{
a Ministério da Saúde. Situação da prevenção e controle das doenças transmissíveis no Brasil. Brasília; 2003. Saúde Brasil 2004: uma análise da situação de saúde. p. 301-37.

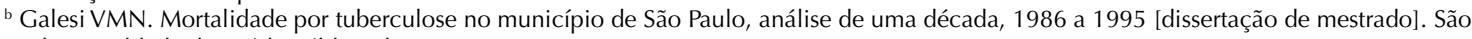
Paulo: Faculdade de Saúde Pública da USP; 1998.

c Secretaria Municipal de Saúde. Centro de Controle de Doenças. Informe TB. 2002;2:1-4.
} 


\section{MÉTODOS}

O município de São Paulo, com cerca de 10 milhões de habitantes, apresenta amplos diferenciais no Índice de Desenvolvimento Humano (IDH), variando dos distritos mais pobres aos mais afluentes de 0,245 a 0,811 . $^{\text {a }}$

Trata-se de estudo descritivo incluindo todos os pacientes, de ambos os sexos, com 15 anos ou mais de idade, residentes no município de São Paulo que evoluíram para óbito por TB, por todas as formas clínicas, como causa básica (códigos A15 a A19 da CID 10), no período de janeiro a dezembro de 2002.

Foram analisadas as seguintes variáveis: sexo, idade, raça/etnia, naturalidade, estado civil, escolaridade, distrito de residência; local de ocorrência do óbito, forma clínica, tipo de serviço onde foi efetuado o diagnóstico, critérios de diagnóstico, intervalo de tempo entre o início dos sintomas e o diagnóstico, duração do tratamento do episódio de TB que evoluiu para o óbito, duração da internação durante a qual ocorreu o óbito, tipo de tratamento, história pregressa de TB, tipo do desfecho do tratamento anterior (cura, abandono), co-morbidades e condições (diabetes, câncer, doença pulmonar obstrutiva crônica, co-infecção TB/HIV, alcoolismo, tabagismo) e notificação ou não do caso ao sistema de vigilância de TB.

A pesquisa utilizou as seguintes fontes: Sistema de Informações de Mortalidade do Município de São Paulo (PROAIM), para identificação dos óbitos de interesse e obtenção das informações disponíveis nos certificados de óbito; prontuários dos hospitais onde ocorreram os óbitos de interesse; Serviço de Verificação de Óbitos (SVO) do Estado de São Paulo para obtenção de resultados de exames anatomopatológicos (macroscópicos); Sistema de Vigilância da Tuberculose do Centro de Controle de Doenças do Município de São Paulo (CCD), para verificação da notificação dos casos e obtenção das informações disponíveis; Fundação Instituto Brasileiro de Geografia e Estatística como fonte de dados populacionais utilizados nos cálculos das taxas de mortalidade. Os dados obtidos na vigilância da TB foram atualizados até setembro de 2003.

O instrumento de coleta de dados foi um formulário pré-codificado no qual foram registradas as informações obtidas nas diferentes fontes consultadas. Foi criado um banco de dados, com dupla digitação, no software EpiInfo versão 6.4.

As taxas de mortalidade segundo o sexo, faixa etária, distrito de residência e naturalidade foram calculadas tomando como numerador os óbitos identificados na pesquisa e como denominador a população com
15 anos ou mais estimada para primeiro de julho de 2002. Os cálculos dos riscos relativos e intervalos de confiança de 95\% (IC 95\%) tomaram o sexo feminino, o grupo de 15 a 29 anos e os naturais do Estado de São Paulo como referência.

Para a elaboração do mapa do município com a apresentação das taxas de mortalidade por distrito utilizamos o programa TabWin. A análise comparativa utilizou o teste do qui-quadrado de Pearson e o exato de Fisher para variáveis categóricas e o teste Kruskal-Wallis para variáveis contínuas. Todos os cálculos foram efetuados usando os softwares estatísticos EpiInfo versão 6.4 e SPSS versão 14 .

A pesquisa foi aprovada pela Comissão de Ética em Pesquisa da Faculdade de Medicina da Universidade de São Paulo.

\section{RESULTADOS}

Foram identificados em 2002, 416 óbitos ocorridos por TB como a causa básica. As formas clínicas mais freqüentes foram a pulmonar $(77,9 \%)$ e a disseminada (17,5\%). Desse total, 44,1\% não haviam iniciado o tratamento, 51,1\% receberam o esquema 1 e $4,8 \%$ o esquema $1 \mathrm{R}$; $20,2 \%$ e $38,5 \%$ foram tratados, respectivamente, por até uma semana ou, no máximo, por período de um mês até a morte.

Entre os tratados, para 82/232 (35,3\%) havia referência de história prévia de $\mathrm{TB}$, dentre os quais 50\% haviam abandonado o tratamento anterior e em 34/82 (41,5\%) a TB ocorrera nos últimos dois anos. Em 30,4\% dos pacientes o diagnóstico de TB foi efetuado após a morte.

Do total dos óbitos 86,0\%, 11,1\% e 2,9\% ocorreram, respectivamente, em hospitais, no domicílio e em via pública. Entre os que no hospital, 20,1\% faleceram nas primeiras 24 horas de internação e 43,1\% em 72 horas. Os critérios de confirmação diagnóstica foram: bacteriológico em 31,8\%, anatomo-patológico (macroscópico) em 38,9\%, clínico-radiológico em 27,2\% e histopatológico em 2,1\%.

Dos 416 óbitos, 19,5\% receberam visita domiciliar. Entre os não notificados à vigilância (n=206; 49,5\%), 187 não iniciaram o tratamento. Para 15 pacientes (3,6\%) o endereço era desconhecido, assim como a identidade em seis deles e nenhum havia sido notificado, sugerindo que fossem moradores de rua.

A mediana da idade dos pacientes foi de 51 anos (16 a 98 anos), sendo de 53 anos para mulheres (17 a 98 anos) e de 50 para homens (16 a 93 anos) ( $>>0,05)$; o sexo masculino respondeu por $75,5 \%$ dos óbitos. De acordo com os certificados de óbito, os pacientes eram

\footnotetext{
a Secretaria do Desenvolvimento, Trabalho e Solidariedade. Índice de Desenvolvimento Humano -IDH. São Paulo; 2002.
} 
brancos (54,7\%), pardos (27,2\%), negros (16,1\%) e amarelos (2,0\%).

Dos 202 óbitos com informação da escolaridade, 51,9\% possuíam menos de quatro anos, $32,7 \%$ de quatro a sete e $15,4 \%$ acima de sete anos de estudo formal. A escolaridade segundo a categorização acima entre nascidos no Estado de São Paulo foi de 37,2\%, 43,0\% e 19,8\%, respectivamente. Entre os nascidos nos demais estados, esses valores são $63,6 \%, 26,1 \%$ e $10,2 \%(p<0,005)$, respectivamente.

A proporção de indivíduos casados (38,2\%; 140/377) foi menor do que aquela formada por solteiros, viúvos, divorciados e sem parceiros fixos, que totalizavam $61,8 \%(233 / 377)(\mathrm{p}<0,001)$.

A taxa de mortalidade por TB encontrada foi de 5,1/100.000 habitantes/ano, sendo de 8,3 para homens e de 2,3 para mulheres, aumentando com a idade (Figura 1). As taxas de mortalidade dos nascidos no Estado de São Paulo, nas regiões Sul e Centro-Oeste e no conjunto das regiões Norte, Nordeste e Sudeste, excluído nesta última o Estado de São Paulo, foram de, respectivamente, 2,5, 3,7 e 6,1 por 100.000 habitantes/ano. Tomando os primeiros como referência, os riscos relativos foram, respectivamente, de 1,48 (IC 95\%: 0,79;2,66) e de 2,48 (IC 95\%: 1,98;3,01).

Em 8/96 (8,3\%) distritos do município não ocorreram óbitos, enquanto que, em 11/96 (11,5\%) as taxas de mortalidade atingiram níveis iguais ou superiores ao dobro da taxa média do município, com o máximo de 34,3/100.000 habitantes/ano. A distribuição das taxas de mortalidade e dos IDH por distrito está ilustrada na Figura 2. A distribuição porcentual dos óbitos mostra que $2,6 \%, 52,2 \%, 34,6 \%$ e $4,1 \%$ residiam em distritos cujo IDH era, respectivamente, inferior a 0,40 , de 0,40 a 0,50 , de 0,51 a 0,69 e superior a 0,69 .

Os pacientes eram também portadores de diabetes (16\%), doença pulmonar obstrutiva crônica (DPOC$19 \%)$, infecção por HIV (11\%), tabagismo (71\%) e alcoolismo (64\%). Dos casos, 84,6\% apresentavam sintomatologia respiratória e 89,3\% perda de peso.

Classificando como não efetivamente tratados aqueles que não iniciaram tratamento ou que foram tratados por até uma semana, não se verificaram diferenças significativas entre tratados e não tratados, segundo sexo, idade, estado civil, escolaridade e etnia $(p>0,05)$ (Tabela 1$)$.

Efetuando a mesma comparação com referência diabetes, câncer, DPOC, co-infecção pelo HIV, presença de sintomas respiratórios a época do diagnóstico, perda de peso, tabagismo e alcoolismo, observa-se maior proporção de pacientes não tratados entre os que apresentavam infecção pelo HIV $(\mathrm{p}<0,005)$ e história de alcoolismo $(\mathrm{p}<0,01)$ (Tabela 2).

\section{DISCUSSÃO}

Desde de 1996, o município de São Paulo tem apresentado um declínio da mortalidade por TB, fato que pode ser atribuído, em parte, à introdução das novas terapias antiretrovirais. Isso tem revertido a tendência de ascensão iniciada em meados dos anos 80 , em função do impacto da co-infecção TB-HIV ${ }^{1,2}$ A despeito desse arrefecimento, a importância da TB como causa de morte no município se mantém, atingindo principalmente a população residente nos distritos com pior IDH, apontando forte influência de fatores socioeconômicos, à semelhança do que ocorre em outras capitais brasileiras. ${ }^{14}$

Tabela 1. Distribuição dos óbitos por tuberculose por todas as formas clínicas, submetidos ou não a tratamento, entre indivíduos com 15 anos ou mais de idade, segundo caracteristicas demográficos e sociais. São Paulo, SP, 2002. N=416

\begin{tabular}{|c|c|c|c|c|c|c|}
\hline \multirow{3}{*}{$\begin{array}{l}\text { Característica } \\
\text { sociodemo- } \\
\text { gráfica }\end{array}$} & \multirow{3}{*}{ Total } & \multicolumn{4}{|c|}{$\begin{array}{l}\text { Pacientes* com } \\
\text { tuberculose submetidos } \\
\text { ao tratamento }\end{array}$} & \multirow[t]{3}{*}{$\mathrm{p}$} \\
\hline & & \multicolumn{2}{|c|}{ Não** } & \multicolumn{2}{|c|}{ Sim } & \\
\hline & & $\mathrm{n}$ & $\%$ & $\mathrm{n}$ & $\%$ & \\
\hline Sexo & & & & & & $>0,05$ \\
\hline Feminino & 102 & 66 & 64,7 & 36 & 35,3 & \\
\hline Masculino & 314 & 201 & 64,2 & 113 & 36,0 & \\
\hline Total & 416 & 267 & 64,3 & 148 & 35,7 & \\
\hline Idade & & & & & & $>0,05$ \\
\hline $15-19$ & 5 & 5 & 100,0 & 0 & 0,0 & \\
\hline $20-49$ & 190 & 121 & 63,7 & 69 & 36,3 & \\
\hline $50-59$ & 84 & 55 & 65,5 & 29 & 34,5 & \\
\hline $60 \mathrm{ou}+$ & 133 & 83 & 62,4 & 50 & 37,6 & \\
\hline Total & 412 & 264 & 64,1 & 148 & 35,9 & \\
\hline Estado civil & & & & & & $>0,05$ \\
\hline Solteiro & 166 & 109 & 65,7 & 57 & 34,3 & \\
\hline Casado & 140 & 81 & 57,9 & 59 & 42,1 & \\
\hline Viúvo & 47 & 30 & 63,8 & 17 & 36,2 & \\
\hline Divorciado & 19 & 12 & 63,2 & 7 & 36,8 & \\
\hline Outros & 4 & 3 & 75,0 & 1 & 25,0 & \\
\hline Total & 376 & 235 & 62,5 & 141 & 37,5 & \\
\hline Escolaridade & & & & & & $>0,05$ \\
\hline$<4$ anos & 105 & 47 & 44,8 & 58 & 55,2 & \\
\hline 4 a 7 anos & 66 & 40 & 60,6 & 26 & 39,4 & \\
\hline$>7$ anos & 31 & 15 & 48,4 & 16 & 51,6 & \\
\hline Total & 202 & 102 & 50,5 & 100 & 49,5 & \\
\hline Etnia & & & & & & $>0,05$ \\
\hline Negra & 65 & 45 & 69,2 & 20 & 30,8 & \\
\hline Branca & 217 & 138 & 63,6 & 79 & 36,4 & \\
\hline Parda & 108 & 72 & 66,7 & 36 & 33,3 & \\
\hline Amarela & 8 & 3 & 37,5 & 5 & 62,5 & \\
\hline Total & 398 & 258 & 64,8 & 140 & 35,2 & \\
\hline
\end{tabular}

* Pacientes com TB que evoluíram para óbito

** Pacientes não tratados ou que receberam tratamento por período inferior a sete dias 
Tabela 2. Distribuição dos óbitos por tuberculose por todas as formas clínicas, submetidos ou não a tratamento, entre indivíduos com 15 anos ou mais de idade, segundo comorbidades, condições e manifestações clínicas. São Paulo, SP, 2002. $\mathrm{N}=416$

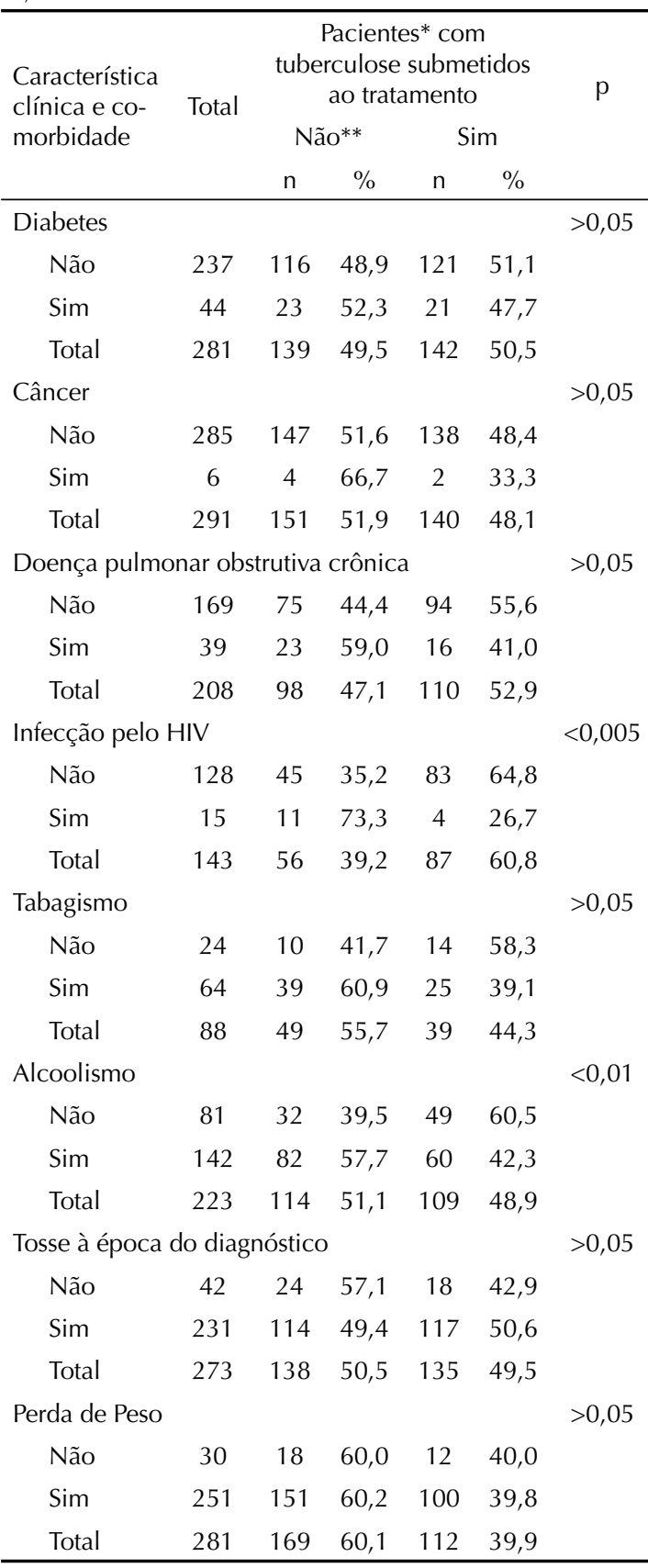

* Pacientes com TB que evoluíram para óbito

** Pacientes não tratados ou que receberam tratamento por período inferior a sete dias

Contudo, os dados apresentados indicam somente parte do problema, pois se os óbitos por TB como causa associada tivessem sido incluídos, a magnitude observada seria expressivamente maior. ${ }^{17}$ Além disso, é possível que os resultados estejam subestimados, pois o número de mortes entre presumíveis moradores de rua foi relativamente baixo, considerando os indicadores sociais do município de São Paulo. Por sua vez, a ausência de óbitos entre pacientes naturais de países latino-americanos contrasta com a importância crescente dessa corrente migratória, originária de regiões com alta prevalência de TB. ${ }^{16}$

Analisando-se as características dos pacientes que evoluíram para óbito, as taxas de mortalidade por sexo e idade encontradas no presente estudo são consistentes com a literatura. ${ }^{14,17,20} \mathrm{O}$ maior risco encontrado entre idosos provavelmente pode ser explicado pela menor imunidade, maior dificuldade de diagnóstico da TB e ao fato das coortes mais velhas terem sido submetidas a maior risco de infecção no passado. ${ }^{22}$

A maior taxa de óbito por TB encontrada entre migrantes de outras regiões do País, explica-se, em parte, pelo fato dessa população ser mais velha, ${ }^{2,5}$ pertencer a segmentos mais pobres da população e ser originária de áreas de maior prevalência de TB. ${ }^{3}$

A alta prevalência de diabéticos, portadores de DPOC, tabagistas e indivíduos com história de alcoolismo indica que fatores biológicos, socioeconômicos e comportamentais influenciaram as condições de vulnerabilidade e contribuíram para a evolução da TB para formas graves e óbito., ${ }^{3,5,12,22}$

A prevalência de co-infecção TB-HIV encontrada é inferior à apontada por estudos realizados na década de 1990,2,20 mas semelhante aos resultados de Oliveira et al, ${ }^{15}$ que mostra diminuição da mortalidade pelos coinfectados, provavelmente resultado da introdução de novos esquemas terapêuticos altamente potentes.

A elevada proporção de casos diagnosticados após o óbito ou não tratados, tratados por menos de um mês, e dos que evoluíram para óbito logo após a internação mostram as dificuldades dos serviços de saúde em identificar e tratar oportunamente parcela significativa dos portadores de TB no município de São Paulo. Tais características permitem classificá-los como potencialmente evitáveis, devendo merecer prioridade nas intervenções de saúde pública.

Somando-se a isso, o predomínio de formas bacilíferas e a pequena parcela de casos identificados em unidades de básicas de saúde, bem como dos que receberam visitas domiciliares, ampliam o risco de transmissão da doença entre os contatos, diminuindo ou neutralizando o impacto das atividades de controle. . $^{8,11}$ À semelhança de outras regiões do País, ${ }^{20}$ cerca de metade dos óbitos eram de casos de TB não notificados, portanto não identificados pelo Programa de Controle de TB até o momento do óbito.

\footnotetext{
a Secretaria do Desenvolvimento, Trabalho e Solidariedade. Índice de Desenvolvimento Humano -IDH. São Paulo; 2002.
} 


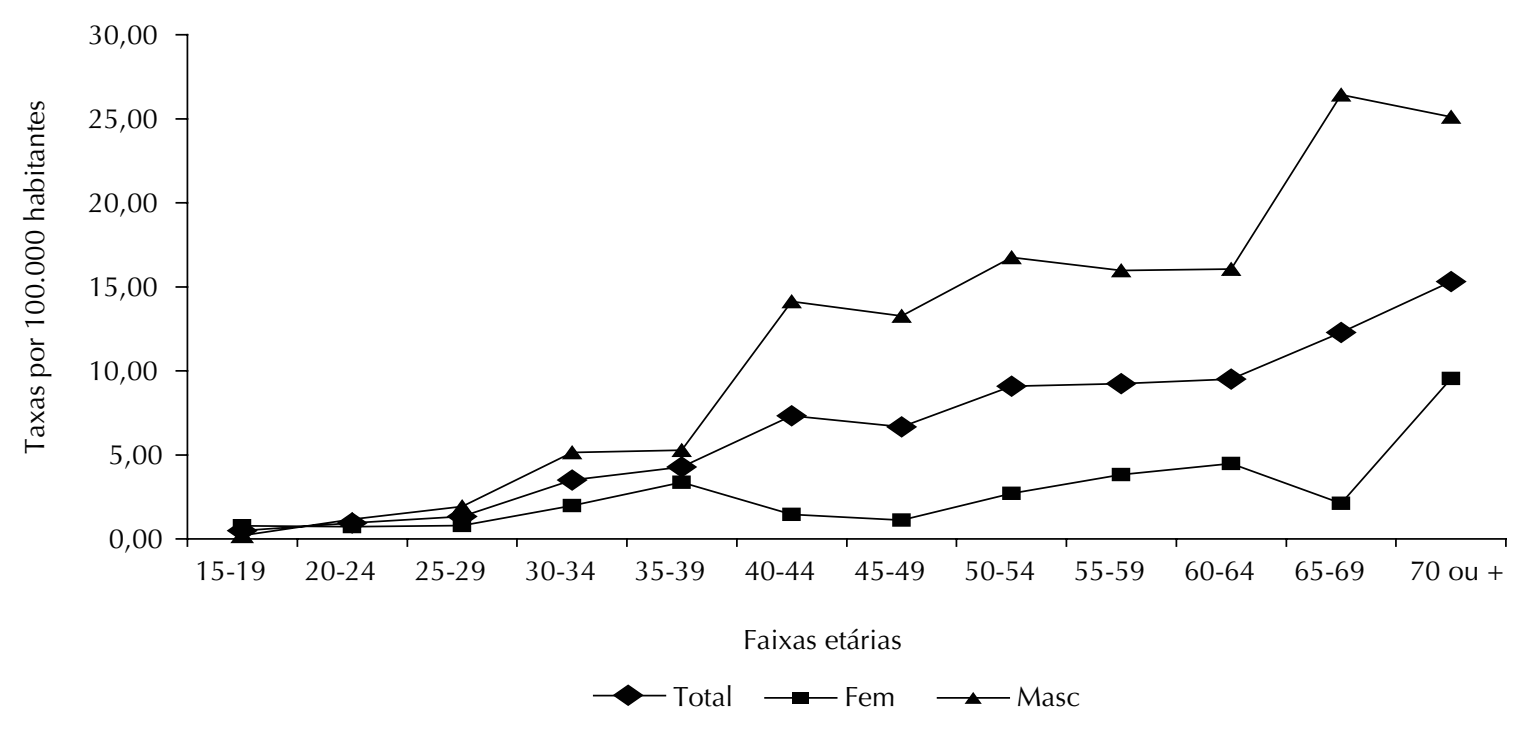

Figura 1. Taxa de mortalidade por tuberculose por todas as formas clínicas entre indivíduos com 15 anos ou mais, residentes no município de São Paulo, segundo faixa etária e sexo. São Paulo, SP, 2002.

A baixa escolaridade dos pacientes que evoluíram para óbito por TB pode ter contribuído para o menor grau de percepção da doença. No entanto, segundo publicação recente, a dificuldade de acesso aos serviços não se mostrou associada à demora no diagnóstico e trata- mento da TB numa capital brasileira. ${ }^{18}$ Tal resultado é consistente com o fato de que no Brasil o acesso ao diagnóstico e ao tratamento da TB é universal e gratuito e o município de São Paulo oferece ampla cobertura pela rede de serviços básicos de saúde.
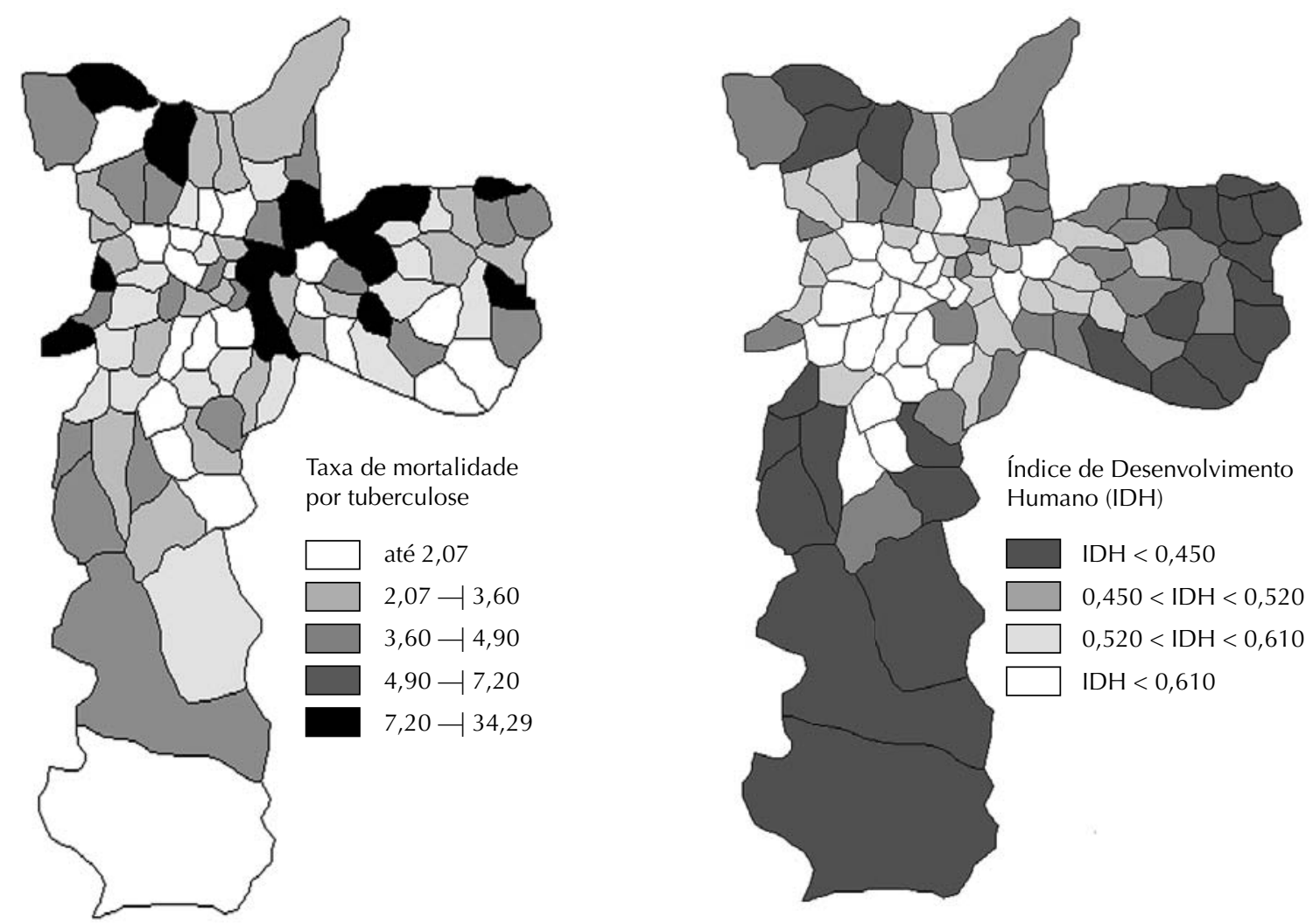

Figura 2. Taxa de mortalidade por tuberculose por todas as formas clínicas como causa básica, entre maiores de 15 anos, por distrito e Índice de Desenvolvimento Humano segundo distrito. São Paulo, SP, 2002. 
O fato de que boa parte dos pacientes apresentava sinais e sintomas característicos da doença indica falha das equipes dos serviços de saúde em suspeitar do diagnóstico de TB em grupos de risco ou em indivíduos que apresentam manifestações clínicas sugestivas.

A elevada proporção de pacientes com história prévia de TB nos últimos dois anos, de abandono desse tratamento, e o fato de parcela dos pacientes ser tratada com esquema $1 \mathrm{R}$, permite-nos supor que parte desses óbitos esteja associada à infecção por Mycobacterium tuberculosis multidroga resistente. ${ }^{5,21}$ Ademais, sugere também o acompanhamento insuficiente de pacientes e de seus comunicantes domiciliares durante e depois do término do tratamento, por período mínimo de dois anos.

A elevada prevalência de infectados pelo Mycobacterium tuberculosis no País não favorece um declínio acentuado da incidência da TB no curto prazo. Porém, os resultados apresentados sugerem que a adoção do tratamento diretamente observado de curta duração (DOTS) com ampla cobertura e prioridade para os distritos de menor IDH, maior concentração de migrantes e com foco em grupos de risco para evolução grave da doença, possibilitará a rápida diminuição da mortalidade por essa causa, à semelhança de países onde a TB constitui igualmente sério problema de saúde pública. ${ }^{6,7,19}$

O acesso universal e gratuito ao diagnóstico e tratamento da TB, a ampla rede de serviços básicos de saúde e a baixa prevalência de infecções por Mycobacterium tuberculosis multidroga resistente no Brasil ${ }^{4,21}$ constituem fatores adicionais que ampliam a condições favoráveis à rápida diminuição da mortalidade por TB.

Para tanto, seria recomendável modificar a estratégia do DOTS no município, que tem visado à cobertura universal dos pacientes com TB, sem um foco especial nos grupos de maior risco. Por outro lado, se fazem necessárias investigações que nos permitam o melhor conhecimento dos preditores de óbito por TB no contexto brasileiro. Em termos operacionais, considerando as dimensões da população, os enormes bolsões de miséria e a elevada incidência da TB, é indispensável que se dimensione adequadamente a infra-estrutura para o diagnóstico, viabilizando a busca sistemática de TB entre sintomáticos respiratórios atendidos pela rede básica de serviços. 


\section{REFERÊNCIAS}

1. Antunes JLF, Waldman EA. Tuberculosis in the twentieth century: time series mortality in São Paulo, Brasil, 1900-97. Cad Saude Publica. 1999;15(3):46376. DOI: 10.1590/S0102-311X1999000300003

2. Antunes JL, Waldman EA. The impact of AIDS, immigration and housing overcrowding on tuberculosis deaths in São Paulo, Brazil, 1994-1998. Soc Sc Med. 2001;52(7):1071-80. DOI: 10.1016/S02779536(00)00214-8

3. Bates I, Fenton C, Gruber J, Lallo D, Lara AM, Squire $\mathrm{SB}$, et al. Vulnerability to malaria, tuberculosis, and HIV/AIDS infection and disease. Part 1: determinants operating at individual and household level. Lancet Infec Dis. 2004;4(5):267-77. DOI: 10.1016/S14733099(04)01002-3

4. Bierranbach $A L$, Gomes $A B$, Noronha EF, Souza MF. Incidência de tuberculose e taxa de cura, Brasil, 2000 a 2004. Rev Saude Publica. 2007;41(Supl 1):24-33. DOI: 10.1590/S0034-89102007000800005

5. Bustamante-Montes LP, Escobar-Mesa A, Borja-Aburto VH, Gómez-Munõz A, Becerra-Posada F.. Predictors of death from pulmonary tuberculosis: The case of Vera Cruz, México. Int J Tuberc Lung Dis. 2000;4(3):208-15.

6. Dye C, Scheele S, Dolin P, Pathania V, Raviglone MC. Consensus statement. Global burden of tuberculosis: estimated incidence, prevalence, and mortality by country. WHO Global Surveillance and Monitoring Project. JAMA. 1999;282(7):677-86. DOI: 10.1001/ jama.282.7.677

7. Dye C, Fengzeng Z, Scheele S, Willians B. Evaluating the impact of tuberculosis control: number of deaths prevented by short-course chemotherapy in China. Int J Epidemiol. 2000;29(3):558-64. DOI: 10.1093/ ije/29.3.558

8. Friedden TR. Can tuberculosis be controlled? Int J Epidemiol. 2002;31(5):894-9. DOI: 10.1093/ ije/31.5.894

9. Lawn SD, Afful B, Acheampong JW. Pulmonary tuberculosis: diagnosis delay in Ghanaian adults. Int J Tuberc Lung Dis. 1998;2(8):635-40.

10. Liefooghe R, Baliddawa JB, Kipruto EM, Vermeire C, De Munynck AO. From their own perspective. A Kenyan community's perception of tuberculosis. Trop Med Int Health. 1997;2(8):809-21. DOI: 10.1046/ j.1365-3156.1997.d01-380.x

11. Lienhardt C. From exposure to disease: the role of environmental factors in susceptibility to and development of tuberculosis. Epidemiol Rev. 2001;23(2):288-301.
12. Mathur P, Sacks L, Auten G, Sall R, Levy C, Gordin F. Delayed diagnosis of pulmonary tuberculosis in city hospitals. Arch Intern Med. 1994;154(3):306-10.

13. Mello Jorge MHP, Gotlieb SLD. As condições de saúde no Brasil. Retrospecto de 1979 a 1995. Rio de Janeiro: FIOCRUZ; 2000.

14. Mota FF, Vieira-da Silva LM, Paim JS, Costa MC. Distribuição espacial da mortalidade por tuberculose em Salvador. Bahia, Brasil. Cad Saude Publica. 2003;19(4):915-22. DOI: 10.1590/S0102311X2003000400014

15. Oliveira HB, Marin-León L, Cardoso JC. Perfil de mortalidade de pacientes com tuberculose relacionada à comorbidade tuberculose-Aids. Rev Saude Publica. 2004;38(4):503-10. DOI: 10.1590/S003489102004000400004

16. Ollé-Goig JE. Patients with tuberculosis in Bolivia: why do they die? Rev Panam Salud Publica. 2000;8(3):1515. DOI: 10.1590/S1020-49892000000800001

17. Santo AH, Pinheiro CE, Jordani MS. Causas múltiplas de morte relacionadas à tuberculose no Estado de São Paulo, 1998. Rev Saude Publica. 2003;37(6):714-21. DOI: 10.1590/S0034-89102003000600005

18. Santos MA, Albuquerque MF, Ximenes RA, LucenaSilva NL, Braga C, Campelo AR, et al. Risk factors for delay treatment in pulmonary tuberculosis in Recife, Brazil. BMC Public Health. 2005;5(1):25. DOI: 10.1186/1471-2458-5-25

19. Suárez P, Watt CJ, Alarcon E, Portocarrero J, Zavala D, Canales R, et al. The dynamics of tuberculosis in response to 10 years of intensive control effort in Peru. J Infec Dis. 2001;184(4):473-8. DOI: $10.1086 / 322777$

20. Selig L, Belo MT, Teixeira EG, Cunha AJ, Brito R, Sanches K, et al. The study of tuberculosis attributed deaths as a tool for disease control planning in Rio de Janeiro, Brazil. Int J Tuberc Lung Dis. 2003:7(9):855-9.

21. Telles MA, Ferrazoli L, Waldman EA, Giampaglia CM, Martins MC, Ueki SY, et al. A population-based study of drug resistance and transmission of tuberculosis in a urban community. Int J Tuberc Lung Dis. 2005;9(9):970-6.

22. Zafran N, Heldal E, Pavlovic S, Vuckovic D, Boe J. Why do our patients die of active tuberculosis in the era of effective therapy? Tuber Lung Dis.1994;75(5):329-33. DOI: 10.1016/09628479(94)90077-9

EA Waldman é apoiado pelo Conselho Nacional de Pesquisas (CNPq; Proc. № 309502/2003-9 - Bolsa Produtividade em Pesquisa (CNPq).

ABP Lindoso foi apoiada pela Coordenação de Aperfeiçoamento do Ensino Superior (Capes; Bolsa de doutorado). 